\title{
Numerical Study of Liquid Metal MHD Flow through a Square Duct under the Action of Strong Transverse Magnetic Field
}

\author{
Dipjyoti Sarma \\ Department of Mathematics \\ Dibrugarh University-786004
}

\author{
G.C.Hazarika \\ Department of Mathematics \\ Dibrugarh University-786004
}

\author{
P.N.Deka \\ Department of Mathematics 3rd \\ Dibrugarh University-786004
}

\begin{abstract}
Numerical solution for steady MHD flow of liquid metal through a square duct under the action of strong transverse magnetic field has been investigated. The walls of the duct are considered to be electrically insulated as well as isothermal. The numerical solutions for velocity and temperature distributions have been obtained by finite difference method. The solutions for different values of Hartmann number and Prandtl number has been analyzed and are presented graphically. The MHD effect on velocity field and temperature field has been predicted in this investigation.
\end{abstract}

\section{Keywords}

MHD flow, liquid metal flow, electrically insulated wall, square duct.

\section{INTRODUCTION}

The flow of liquid metals through ducts under transverse magnetic field arises in many industrial applications such as casting of steel, aluminum reduction etc. The liquid metal flow across the intense plasma confinement field arises in fusion engineering for tritium breeder and coolant blanket design. Besides these, in number of cases, concerning engineering applications, flow of liquid metal across magnetic field may be involved. In view of current trend of industrial applications, the study of problems concerning liquid metal flow through duct under action of transverse magnetic field are studied with great interest by the engineers and physicists during last few decades. In fusion blanket design, it is required to understand the flow of liquid metal in channels or pipes under the action of very intense magnetic field. Such a case of duct flow is associated with sharp pressure drop phenomena and heat transfer and other processes. To understand behavior of such flow, appropriate knowledge of MHD relationship will be necessary.

MHD flow problem of liquid metal through a duct under the action of transverse magnetic field was first considered by Hartmann and Lazarus [1]. They considered flow of mercury as a conducting fluid in pipes of different cross sections experimentally and in their investigations, the influence of transverse field in such a flow was elucidated. Theoretical investigation for electrically conducting flow through pipes was subsequently considered by Shercliff[2, 3].In these investigations Shercliff, studied electrically conducting viscous fluids in pipes under the action of transverse magnetic field and improved results were obtained. Further, Shercliff considered the laminar motion of a conducting liquid at high Hartmann number in non-conducting pipes of arbitrary cross section under uniform transverse magnetic field. Gupta and Singh [4] obtained an exact solution for the case of unsteady MHD flow in a circular pipe having insulated wall under the action of a uniform magnetic field parallel to a diameter of the cross section. Singh and Lal [5] obtained numerical solution for steady MHD flow in a triangular pipe of non-conducting wall under the action of transverse magnetic field perpendicular to a side. Walker [6] studied the liquid metal flow in a circular pipe with a thin metal wall. Kim and Abdou [7] developed a numerical algorithm to provide a fully detailed flow field in liquid metal MHD flow with a relatively large Hartmann number and interaction parameter. Kunugi et.al.[8] developed a new computer code (KAT)with the capability to model laminar liquid metal flow and heat transfer in relatively complex geometries. L Bhuler [9] studied the MHD flow quantities in channels with arbitrary cross section and a nearly arbitrary orientation with respect to a strong variable magnetic field. Smolentsev [10] considered two mathematical models for MHD flows in a fusion reactor blanket where first one described fully-developed flows and the second governs non-uniform and non-steady flows. Barrett [11] determines the flow in a straight channel under a variety of wall conductivity conditions when a uniform magnetic field is imposed to the flow direction by using finite element methods. Celik [12] solve the MHD flow equations in a rectangular duct in presence of transverse external oblique magnetic field by Chebyshev collocation method [13] AlKhawaja et. al. [14] studied the flow of liquid metal in a square duct under the action of strong magnetic field perpendicular to a side of duct.

In this paper the fluid flow as well as the heat transfer problem of liquid metal flow through a square duct under the action of strong transverse magnetic field has been investigated. An improved solution for flow and temperature fields for different values of Hartmann numbers and for values of Prandtl numbers within allowable range for liquid metal flows has been obtained. This study has an intensity to include heat transfer effect in such duct flows.

\section{FORMULATION OF THE PROBLEM}

In this problem we consider the steady motion of incompressible liquid metal through a square duct under the 


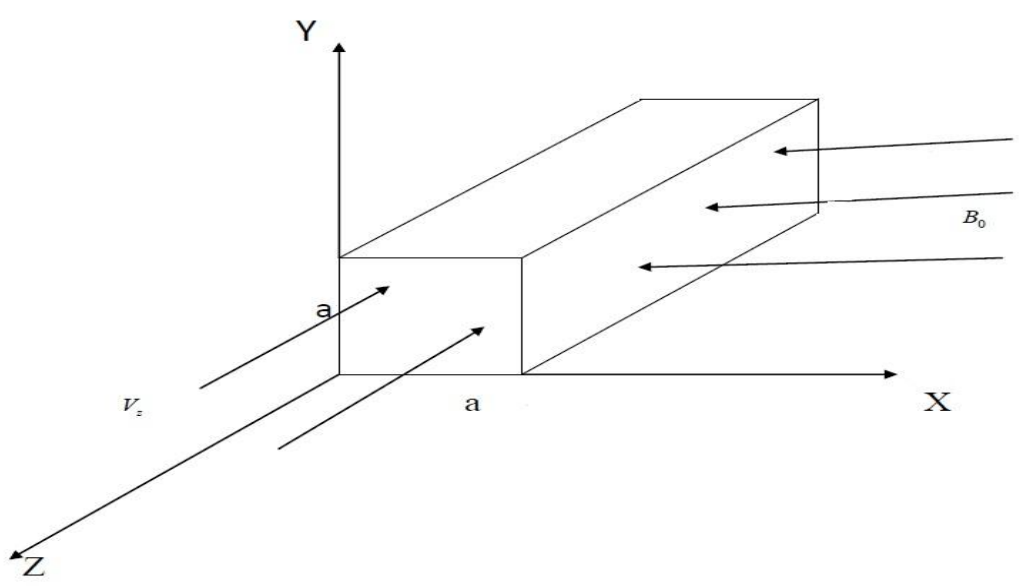

Fig 1: Geometry of the problem

action of strong transverse magnetic field applied perpendicular to two opposite walls of the duct. In this study following assumptions are considered:

(i)All fluid properties are constant and independent of the temperature.

(ii)The flow and heat transfer are fully developed.

Under these assumptions, the velocity, magnetic field and temperature will be of the form

$\vec{V}=\left[0,0, V_{z}(x, y)\right]$

$\vec{B}=\left[B_{0}, 0, B_{z}(x, y)\right]$

$T=T(x, y)$

The governing equations of the flow are Equation of Continuity:

$$
\nabla \cdot \vec{V}=0
$$

Momentum Equation:

$$
\rho\left[\frac{\partial \vec{V}}{\partial t}+(\vec{V} \cdot \nabla) \vec{V}\right]+\nabla p=\vec{J} \times \vec{B}+\mu \nabla^{2} \vec{V}
$$

Energy Equation:

$$
\rho C_{p}\left[\frac{\partial T}{\partial t}+(\vec{V} . \nabla) T\right]=\nabla \cdot(k \nabla T)+\mu \phi+\frac{J^{2}}{\sigma}
$$

Ohms law:

$$
\vec{J}=\sigma[\vec{E}+\vec{V} \times \vec{B}]
$$

Amperes law

$\nabla \times \vec{B}=\mu_{e} \vec{J}$
Faradays law (for steady flow)

$\nabla \times \vec{E}=-\frac{\partial \vec{B}}{\partial t}=0$

Using Equation (5) in Equation (2) for this velocity and assuming

$\frac{\partial p}{\partial z}=-P($ constant $)$

We finally obtain

$\mu \nabla^{2} V_{z}+\frac{B_{0}}{\mu_{e}} \frac{\partial B_{z}}{\partial x}=-P$

Using equation (4) and (6), equation (2) reduces to

$\lambda \nabla^{2} B_{z}+B_{0} \frac{\partial V_{z}}{\partial x}=0$

Equation (3) now reduces to

$k\left(\frac{\partial^{2} T}{\partial x^{2}}+\frac{\partial^{2} T}{\partial y^{2}}\right)+\mu\left[\left(\frac{\partial V_{z}}{\partial x}\right)^{2}+\left(\frac{\partial V_{z}}{\partial y}\right)^{2}\right]+$

$\frac{1}{\sigma \mu_{e}^{2}}\left[\left(\frac{\partial B_{z}}{\partial x}\right)^{2}+\left(\frac{\partial B_{z}}{\partial y}\right)^{2}\right]=0$

The non dimensional quantities used in this problem are defined as follows :

$\theta=\frac{T-T_{a}}{\Delta T}, x^{*}=\frac{x}{L}, B^{*}=\frac{B_{z}}{B_{0}}, y^{*}=\frac{y}{L}$,

$\mathrm{V}^{*}=\frac{V_{z}}{V_{0}}, V_{0}=\frac{p L^{2}}{\mu}, M=B_{0} L\left(\frac{\sigma}{\mu}\right)^{1 / 2}$

$R_{m}=\mu_{e} \sigma V_{0} L, E_{c}=\frac{V_{0}^{2}}{C_{p} \Delta T}, P_{r}=\frac{\mu C_{p}}{k}$ 
The non-dimensional equations are

$\frac{\partial^{2} V}{\partial x^{2}}+\frac{\partial^{2} V}{\partial y^{2}}+\frac{M^{2}}{R_{m}} \frac{\partial B}{\partial x}=-1$

$\frac{\partial^{2} B}{\partial x^{2}}+\frac{\partial^{2} B}{\partial y^{2}}+R_{m} \frac{\partial V}{\partial x}=0$

$\frac{\partial^{2} \theta}{\partial x^{2}}+\frac{\partial^{2} \theta}{\partial y^{2}}+P_{r} E_{c}\left[\left(\frac{\partial V}{\partial x}\right)^{2}+\left(\frac{\partial V}{\partial y}\right)^{2}\right]+$

$\frac{M^{2} E_{c} P_{r}}{R_{m}{ }^{2}}\left[\left(\frac{\partial B}{\partial x}\right)^{2}+\left(\frac{\partial B}{\partial y}\right)^{2}\right]=0$

The boundary conditions considered are:

$V=0$ (from no slip condition $)$

$B=0$ (for externally insulated surface $)$

$\theta=0$ (for isothermal surface)

Here it is observed that Equation (11) - (13) are coupled nonlinear equations which are to be solved using prescribed boundary conditions. In view of complexities in seeking closed form solutions, numerical solutions are considered. These equations are expressed in finite difference scheme followed by Schmidit[15]. The mesh system considered involves grid points with uniform spacing of $\Delta x=0.0625$ and $\Delta y=0.025$. The resulting finite difference equation for Equation (11)-.(13) are given as follows

$$
\begin{aligned}
V_{i, j}= & C_{1}\left[k^{2}\left(V_{i-1, j}+V_{i+1, j}\right)+h^{2}\left(V_{i, j-1}+V_{i, j+1}\right)\right]+ \\
& C_{2}\left(B_{i+1, j}-B_{i, j}\right)+C_{3} \\
B_{i, j}= & C_{1}\left[k^{2}\left(B_{i-1, j}+B_{i+1, j}\right)+h^{2}\left(B_{i, j-1}+B_{i, j+1}\right)\right]+ \\
& C_{4}\left(V_{i+1, j}-V_{i, j}\right) \\
\theta_{i, j}= & C_{1}\left[k^{2}\left(\theta_{i-1, j}+\theta_{i+1, j}\right)+h^{2}\left(\theta_{i, j-1}+\theta_{i, j+1}\right)\right]+ \\
& C_{5}\left(V_{i+1, j}-V_{i, j}\right)^{2}+C_{6}\left(V_{i, j+1}-V_{i, j}\right)^{2}+ \\
& C_{7}\left(B_{i+1, j}-B_{i, j}\right)^{2}+C_{8}\left(B_{i, j+1}-B_{i, j}\right)^{2}
\end{aligned}
$$

Where

$$
\begin{aligned}
& C_{1}=\frac{1}{2\left(h^{2}+k^{2}\right)}, C_{2}=\frac{M^{2} h k^{2}}{2\left(h^{2}+k^{2}\right) R_{m}}, \\
& C_{3}=\frac{h^{2} k^{2}}{2\left(h^{2}+k^{2}\right)}, C_{4}=\frac{h k^{2} R_{m}}{2\left(h^{2}+k^{2}\right)}, \\
& C_{5}=\frac{k^{2} P_{r} E_{c}}{2\left(h^{2}+k^{2}\right)}, C_{6}=\frac{h^{2} P_{r} E_{c}}{2\left(h^{2}+k^{2}\right)} \\
& C_{7}=\frac{k^{2} P_{r} E_{c} M^{2}}{2\left(h^{2}+k^{2}\right) R_{m}^{2}}, C_{8}=\frac{h^{2} P_{r} E_{c} M^{2}}{2\left(h^{2}+k^{2}\right) R_{m}^{2}}
\end{aligned}
$$

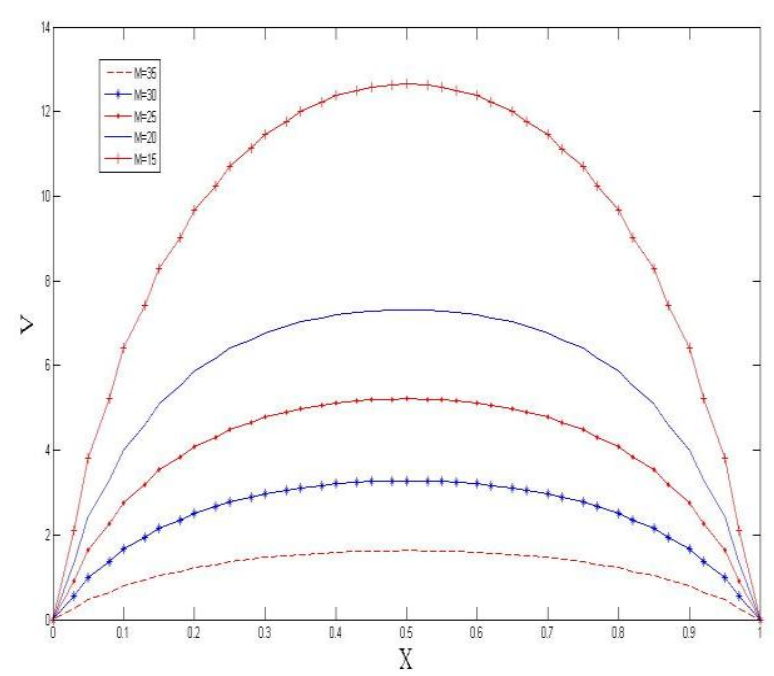

Fig 2: Variation of velocity profile for different Hartmann Number

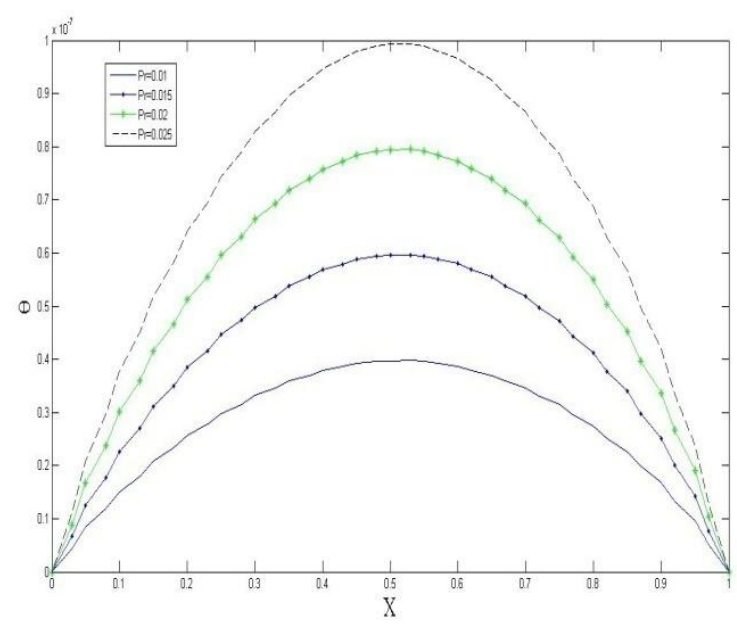

Fig 3: Variation of temperature profile for different Prandtl Number

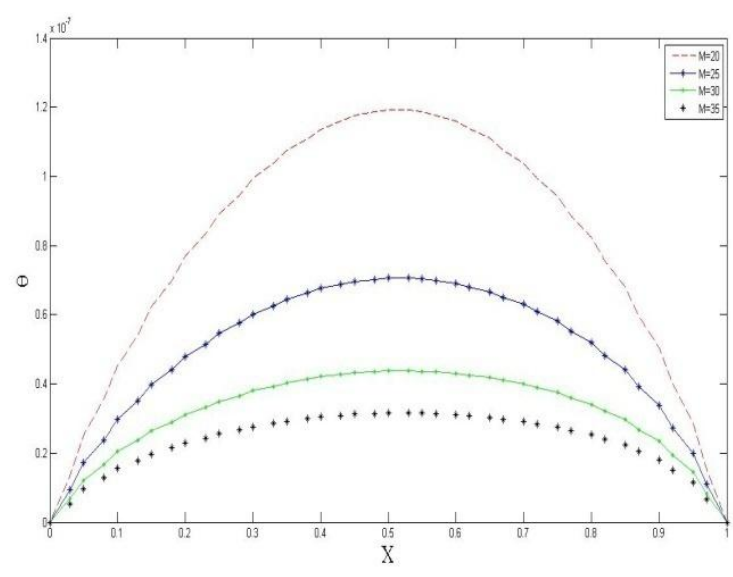

Fig 4: Variation of temperature profile for different Hartmann Number 


\section{RESULT AND DISCUSSION}

In this problem liquid metal flow through a duct aligned to the horizontal direction is considered and accordingly buoyancy force is not affecting the flow. The transverse magnetic field reduces the frictional force out from the. boundary and flow is enhanced as the intensity of external magnetic field is increased. But very strong transverse magnetic field produces Lorentz's force $\vec{J} \times \vec{B}$ opposing the flow and creating large pressure loss. Since in the present case walls of the duct are assumed to be electrically insulated, the development of induced current associated with strong transverse field cannot be intensified itself. In this investigation of MHD duct flow, Hartmann numbers of values of order $10^{2}$ have been considered In such environment flow opposing pressure drop phenomena is not prominent.

The velocity distribution plotted in Fig 2 for different values of Hartmann number shows the effect of increased Hartmann number on flow field. In conditions prevails in a high -field tokamak reactor, MHD duct flow is associated with pressure drop phenomena. However, in such case "core flow approximation" [16] can be applied in which inertia and viscous terms are omitted. In such assumption, much simple numerical solutions are possible. Very high Hartmann flows are also subjected to corrections due to side layers. In subsequent study various aspects of very high Hartmann flow may be investigated.

In this result it is observed that the boundary is narrower along with the increase of values of Hartmann number. In case calculations have shifted with higher order Hartmann number, Hartmann layer will be much narrower than the observed result in this problem. For such a case the effect the interaction parameter, $\mathrm{N}$ the ratio of electromagnetic force and inertia force $[17,18,19]$ is to be included. The temperature variation in the flow condition also being plotted. Under this flow condition the variation of temperature field has been shown in plotting (2) and (3). In plot (2) variation of temperature field has been shown against the values of Prandtl number and in plotting (3) variation of temperature field has been shown against the values of Hartmann number. From the plotting given in Fig 4 it is found that along with the increase of Hartmann number temperature increases. Further, from plotting given in Fig 3 it is observed that increase in Prandtl numbers result in increase in temperature of the flow. Here the variation of Prandtl number only within the allowable range 0.004 to 0.03 [20] are considered. It has been noted that the results obtained in this numerical investigation, in essence, do not contradict the numerical solutions for duct flow under similar condition but for simpler models[12,14].

\section{ACKNOWLEDGMENTS}

This work was supported by University Grant Commission, India under grant number39-44/2010(SR).

\section{REFERENCES}

[1] Hartmann,J. and Lazarus,F. 1937. Experimental investigations on the flow of mercury in a homogeneous msagnetic field. K.Dan.Vidensk.Selsk.Mat.Fys.Medd. vol 15(6).1-45.

[2] Shercliff,J.A. 1953. Steady motion of conducting fluids in pipes under transverse magnetic field. Mathematical Proc. of the Cambridge Philosophical Society.vol 49(1).136-144.
[3] Shercliff, J.A.1962. Magnetohydrodynamic pipe flow Part 2, High Hartmann number. Journal of Fluid Mechanics.vol 13(4).513-518.

[4] Gupta, S.C. and Singh, B. 1970. Unsteady Magnetohydrodynamic Flow in a circular pipe under a Transverse Magnetic Field. Physics of Fluids.vol 13(2).346-352.

[5] Singh,B. and Lal,J.1978.Magnetohydrodynamic axial flow in a triangular pipe under transverse magnetic field. Indian Journal of Pure \& Applied Mathematics.vol 9(2).101-115.

[6] Walker, S. 1986. Liquid metal flow in a thin conducting pipe near the end of a region of uniform magnetic field. Journal of Fluid Mechanics.vol 167.199-217.

[7] Kim, C.N. and Abdou, M.A.1989.Numerical Method for Fluid Flow and Heat Transfer in Magnetohydrodynamic Flow. Fusion Technology.vol 15.1163-1168.

[8] Kunugi, T., Tillack, M.S. and Abdou, M.A.1991.Analysis of Liquid Metal MHD Fluid Flow and Heat Transfer using the KAT Code. Fusion Technology.vol 19.1000-1005.

[9] Buhler,L. 1991. Liquid metal flow in arbitrary thinwalled channels under a strong transverse variable magnetic field. Fusion Engineering and Design.vol $17.215-220$

[10] Smolentsev,S.Yu. 1999. Mathematical models for magnetohydrodynamic flows in a fusion reactor blanket. Plasma Devices and Operations.vol 7.231-241.

[11] Barrett, K.E.2001.Duct flow with a transverse magnetic field at high Hartmann numbers. International Journal for numerical methods in engineering.vol 50(8).1893-1906.

[12] Celik,I. 2011. Solutions of magnetohydrodynamic flow in a rectangular duct by Chebyshev Collocation method. International Journal for Numerical methods in fluids.vol 66(10).1325-1340.

[13] Kesan,C. 2003.Chebyshev polynomial solutions of second order linear partial differential equations. Applied Mathematics and Computations.vol 134(1).109-124.

[14] Al-Khawaja,M. 2010 Matlab-Modelling, programming and simulations. Sciyo.

[15] Jain, M.K., Iyengar, S.R.K. and Jain, R.K. 1994 Computational Methods for Partial Differential Equations. Wiley Eastern Limited.

[16] Tillack, M.S.1989. Liquid Metal Magneto hydrtodynamics. Kluwer Academic Publishers.

[17] Moreau, R. 1990. Magnetohydrodynamics. Kluwer Academic Publishers.

[18] McCarthy, K.A. and Abdou, M.A.1991. Analysis of liquid metal MHD flow in multiple adjacent ducts using an iterative method to solve the core flow equations. Fusion Engineering and Design. vol 13. 363-380.

[19] Kim,C.N. Hadid Ali H. and Abdou, M.A. 1989 Development of a computational method for the ful solution of MHD flow in Fusion Blankets. Fusion Engineering and Design. Vol 8. 265-270.

[20] Thirumaleshwar, M. 2006. Fundamental of Heat and Mass Transfer. Dorling Kindersley Pvt.Ltd. 